\title{
Mortality due to systemic mycoses as a primary cause of death or in association with AIDS in Brazil: a review from 1996 to 2006
}

\author{
Marli Prado', Marcelo Barbosa da Silva², Ruy Laurenti', Luiz R Travassos ${ }^{3}$, Carlos P Taborda $^{2}+$ \\ ${ }^{2}$ Instituto de Ciências Biomédicas, Departamento de Microbiologia e Laboratório de Micologia Médica - LIM53 - Hospital de Clínicas \\ da Faculdade de Medicina 'Departamento de Epidemiologia, Escola de Saúde Pública, Universidade de São Paulo, São Paulo, SP, Brasil \\ ${ }^{3}$ Departamento de Microbiologia, Imunologia e Parasitologia, Universidade Federal de São Paulo, São Paulo, SP, Brasil
}

Deaths caused by systemic mycoses such as paracoccidioidomycosis, cryptococcosis, histoplasmosis, candidiasis, aspergillosis, coccidioidomycosis and zygomycosis amounted to 3,583 between 1996-2006 in Brazil. When analysed as the underlying cause of death, paracoccidioidomycosis represented the most important cause of deaths among systemic mycoses ( 51.2\%). When considering AIDS as the underlying cause of death and the systemic mycoses as associated conditions, cryptococcosis (50.9\%) appeared at the top of the list, followed by candidiasis $(30.2 \%)$, histoplasmosis (10.1\%) and others. This mortality analysis is useful in understanding the real situation of systemic mycoses in Brazil, since there is no mandatory notification of patients diagnosed with systemic mycoses in the official health system.

Key words: mortality - systemic mycoses - AIDS - deep mycoses

In Brazil, epidemiological research on mortality is carried out by the Mortality Information System (MIS), created in 1976 with the implementation throughout the national territory of a standardised death certificate (DC). Even though data from the MIS are abundant and trustworthy for mortality studies, some states in Brazil still have uncertain coverage and information quality, with high rates of under-registration of deaths. Underregistration has primarily been attributed to the custom of performing burials without a formal DC, which generally occurs in poor and rural areas. Studies from the Ministry of Health showed that death information in the MIS covered $94.6 \%$ of deaths in the South, $90.9 \%$ in the Southeast, $87.3 \%$ in the Central West and $74.6 \%$ in the North and Northeast of Brazil, where under-registration is more common (MS 2004, 2005).

Since fungal diseases are not bound by mandatory notification in the Brazilian Health System, the real situation of systemic mycoses in Brazil is difficult to assess. Brazil has a surface area of $8,547,403.5 \mathrm{~km}^{2}$, corresponding to approximately $47 \%$ of South America (IBGE 2000). Statistics based on skin tests or serological assays showed differences in the prevalence of infection in endemic areas as well as differences attributed to the kind of antigen used in the tests. Indeed, the utilisation of purified or crude antigens for the diagnosis of fungal infections may give rise to different results since crude preparations may increase cross-reactivity in sera from patients with other diseases.

Financial support: CPT and LRT were supported by FAPESP (07/07588-2, 06/50634-2 and 05/02776-0), CNPq (470636/2007-6).

+Corresponding author: taborda@usp.br

Received 8 September 2008

Acceppted 3 March 2009
The present study aimed at evaluating the mortality rates attributable to the most important systemic mycoses in Brazil, namely paracoccidioidomycosis, histoplasmosis, cryptococcosis, candidiasis, zygomycosis and aspergillosis, and their association with environmental conditions and immunosuppressive diseases like AIDS. We have mapped the spatial distribution of deaths, sex, age and other factors associated with mortality from systemic mycoses in Brazil (MS 2004, 2005).

\section{METHODS}

The mortality data for systemic mycoses were scored based on historical records from the past 11 years, from 1996-2006, focusing on the death database maintained by the Ministry of Health and made available on the website www.datasus.gov.br (MS 2005). For 2006, preliminary data available were obtained from the General Coordination of Information and Epidemiological Analyses of the Department of Analysis of Health Situations, which is under the Secretary of Health Vigilance of the Brazilian Ministry of Health. Census data, demographic projections and territorial area data were obtained from the Brazilian Institute of Geography and Statistics and are available on the website www.ibge.gov.br. During this period, the underlying causes of death were classified according to the 10th revision of the International Classification of Diseases, ICD-10.

In ICD-10, mycoses are included in categories B35 to B49 in Chapter 1 (Certain infectious and parasitic diseases). For systemic mycoses, the following categories were selected: B37-candidiasis, B38-coccidioidomycosis, B39-histoplasmosis, B40-blastomycosis, B41-paracoccidioidomycosis, B44-aspergillosis, B45cryptococcosis and B46-zygomycosis. The codes B-40 and B-41 were grouped, since the designation "paracoccidioidomycosis" for South American blastomycosis was accepted in 1971 in Medellín, Colombia, during the International Meeting on Paracoccidioidomycosis. 
In order to reduce confounding sources (age, geographic area and time), we used standardised rates obtained via a direct method, using the 2000 census of the Brazilian population as the reference; different populations in this study cannot be compared with each other because there are differences in the age structure (Rumel 1988, Laurenti et al. 2005). The indicator used for the values, evolution and comparison of mortality was the standard rate of mortality per gender and age, using the age structure of the 2000 census of the Brazilian population as the reference. The mortality coefficient of systemic mycoses was calculated per million inhabitants and the estimated risk of death was calculated per 100,000 inhabitants for other infectious and parasitic diseases. We also calculated a ratio of standardised mortality rates comparing rates at the end (2005-2006) and the beginning (1996-1998) of the study period.

To evaluate the association between systemic mycoses and AIDS (categories B20-B24), the mention of any systemic mycosis anywhere in Part I and Part II of the DC was considered. The indicators utilized were the frequency (\%) of mentions of systemic mycoses in the total number of deaths in which the underlying cause was AIDS, and the relation with the number of mentions of systemic mycoses in every 1,000 AIDS deaths when the basic cause of death was AIDS.

The parameters selected for this study were sex, age, occupation and underlying cause of death in any geographic location, state or region in the country. DC for which any selected parameter was missing was discarded; the number of such certificates is indicated at the bottom of the Tables.

\section{RESULTS}

In Brazil, infectious and parasitic diseases (Chapter I, ICD-10) were responsible for 517,058 deaths from 19962006. We excluded 2,683 deaths for which the records did not include age or that were otherwise not adequately filled out, yielding a total of 514,375 death records analysed for this study. In the first three years of the study, the annual average number of deaths was 49,256 , while it was 45,164 in the second three-year period of the study, 45,781 in the third three-year period and 46,888 in the last two years. The mortality rate from infectious and parasitic diseases was stable, increasing only from 28.25-28.88 per 100,000 people (MS 2004, 2005).

Among infectious and parasitic diseases, in the period from 1996-2006, systemic mycoses were the underlying cause for 3,583 annual deaths in 1,314 (23.6\%) of 5,560 Brazilian municipalities. The total number of deaths and the annual averages were as follows: period 1 (1996-1998): 952 deaths, annual average of 316 deaths; period 2 (1999-2001): 936 deaths, annual average of 312 deaths; period 3 (2002-2004): 1,034 deaths, annual average of 344 deaths. In period 4 (2005 and preliminary data from 2006) there were 661 deaths and an annual average of 331 deaths. A similar process was used for demographic data. Systemic mycoses ranked as the 10th cause of death among infectious and parasitic diseases (Chapter I, ICD-10), below other diseases such as hanseniasis, pertussis, sepsis, HIV, intestinal infections, tuberculosis, protozoan diseases like malaria and Chagas disease and viral hepatitis (MS 2004, 2005).

Deaths due to systemic mycoses - For the 3,583 deaths due to systemic mycoses that occurred in the decade from 1996-2006, paracoccidioidomycosis (including blastomycosis) was the most frequently mentioned mycosis, with an annual average of 171 deaths $(53.9 \%)$ at the beginning of the study period and 148 deaths $(44.6 \%)$ at the end. Other systemic mycoses showed increasing numbers of deaths in the same period. The annual average number of deaths from cryptococcosis increased from $78(24.5 \%)$ at the beginning of the study period to $89(26.8 \%)$ at the end. Candidiasis was responsible for an average 39 annual deaths $(12.2 \%)$ at the beginning of the study period and 54 (16.3\%) deaths at the end. A similar trend of increasing average annual deaths was observed for the other mycoses (Table I).

Deaths and geographic distribution of the most important systemic mycoses - The four most important causes of death among systemic mycoses were analysed separately by period (1-4) and by region and state.

TABLE I

Number and frequency of deaths per systemic mycosis (3C - ICD - 10). Brazil, annual average, 1996-2006 ${ }^{a}$

\begin{tabular}{|c|c|c|c|c|c|c|c|c|}
\hline \multirow[b]{2}{*}{ Systemic mycosis (ICD-10) } & \multicolumn{2}{|c|}{$\begin{array}{c}\text { Annual average } \\
1996-1998\end{array}$} & \multicolumn{2}{|c|}{$\begin{array}{c}\text { Annual average } \\
\text { 1999-2001 }\end{array}$} & \multicolumn{2}{|c|}{$\begin{array}{l}\text { Annual average } \\
\text { 2002-2004 }\end{array}$} & \multicolumn{2}{|c|}{$\begin{array}{c}\text { Annual average } \\
2005-2006^{a}\end{array}$} \\
\hline & $\mathrm{n}$ & $\%$ & $\mathrm{n}$ & $\%$ & $\mathrm{n}$ & $\%$ & $\mathrm{n}$ & $\%$ \\
\hline Blastomycosis + Paracoccidioidomycosis & 171 & 53.9 & 173 & 55.3 & 175 & 50.9 & 148 & 44.6 \\
\hline Cryptococcosis & 78 & 24.5 & 76 & 24.3 & 81 & 23.5 & 89 & 26.8 \\
\hline Candidiasis & 39 & 12.2 & 36 & 11.5 & 51 & 14.9 & 54 & 16.3 \\
\hline Histoplasmosis & 15 & 4.8 & 10 & 3.2 & 12 & 3.6 & 19 & 5.6 \\
\hline Aspergillosis & 12 & 3.8 & 13 & 4.1 & 18 & 5.3 & 17 & 5.0 \\
\hline Zygomycosis & 2 & 0.5 & 4 & 1.3 & 4 & 1.3 & 3 & 0.9 \\
\hline Coccidioidomycosis & 1 & 0.3 & 1 & 0.3 & 2 & 0.6 & 3 & 0.8 \\
\hline Total & 317 & 100.0 & 312 & 100.0 & 345 & 100.0 & 331 & 100.0 \\
\hline
\end{tabular}

a: preliminary data for 2006 . 
TABLE II

Mortality rate TBM and TPM by paracoccidioidomycosis (underlying cause - codes B41.0-B41.9 - Chapter I - some parasitic and infectious disease - ICD 10) in regions and states of Brazil. Period from 1996-2006 ${ }^{a}$

\begin{tabular}{|c|c|c|c|c|c|c|c|c|c|c|c|c|}
\hline \multirow{2}{*}{$\frac{\text { Period }}{\text { Regions and states }}$} & \multicolumn{3}{|c|}{$\begin{array}{c}\text { Average } \\
1996-1998 \text { (I) }\end{array}$} & \multicolumn{3}{|c|}{$\begin{array}{c}\text { Average } \\
1999-2001 \text { (II) }\end{array}$} & \multicolumn{3}{|c|}{$\begin{array}{c}\text { Average } \\
2002-2004 \text { (III) }\end{array}$} & \multicolumn{3}{|c|}{$\begin{array}{c}\text { Average } \\
2005-2006^{a}(\mathrm{IV})\end{array}$} \\
\hline & deaths & TBM & TPM & deaths & TBM & TPM & deaths & TBM & TPM & deaths & TBM & TPM \\
\hline North & 11 & 1.0 & 0.9 & 19 & 1.5 & 1.5 & 25 & 1.8 & 1.9 & 17 & 1.1 & 1.4 \\
\hline Rondônia & 5 & 3.7 & 3.1 & 9 & 6.9 & 6.9 & 12 & 8.2 & 8.7 & 6 & 3.9 & 4.8 \\
\hline Acre & 1 & 2.7 & 2.3 & 2 & 3.6 & 3.6 & 3 & 5.6 & 6.0 & 1 & 1.5 & 1.8 \\
\hline Amazonas & $*$ & $*$ & $*$ & $*$ & $*$ & $*$ & 0 & 0.1 & 0.1 & $*$ & $*$ & $*$ \\
\hline Roraima & $*$ & $*$ & $*$ & $*$ & $*$ & $*$ & 1 & 1.9 & 2.1 & 1 & 1.3 & 1.5 \\
\hline Para & 3 & 0.5 & 0.4 & 5 & 0.8 & 0.8 & 5 & 0.8 & 0.8 & 6 & 0.8 & 1.0 \\
\hline Amapá & $*$ & $*$ & $*$ & $*$ & $*$ & $*$ & 0 & 0.6 & 0.7 & $*$ & $*$ & $*$ \\
\hline Tocantins & 3 & 2.5 & 2.3 & 3 & 2.6 & 2.6 & 3 & 2.4 & 2.6 & 4 & 3.0 & 3.6 \\
\hline Northeast & 6 & 0.1 & 0.1 & 4 & 0.1 & 0.1 & 8 & 0.2 & 0.2 & 4 & 0.1 & 0.1 \\
\hline Maranhão & 1 & 0.2 & 0.2 & 1 & 0.2 & 0.2 & 4 & 0.6 & 0.6 & 2 & 0.3 & 0.4 \\
\hline Piauí & 0 & 0.1 & 0.1 & 0 & 0.1 & 0.1 & 1 & 0.3 & 0.4 & $*$ & $*$ & $*$ \\
\hline Ceara & 1 & 0.1 & 0.1 & $*$ & $*$ & $*$ & $*$ & $*$ & $*$ & $*$ & $*$ & $*$ \\
\hline Rio Grande do Norte & 1 & 0.3 & 0.3 & $*$ & $*$ & $*$ & $*$ & $*$ & $*$ & 1 & 0.2 & 0.2 \\
\hline Paraíba & $*$ & $*$ & $*$ & 0 & 0.1 & 0.1 & 1 & 0.3 & 0.3 & $*$ & $*$ & $*$ \\
\hline Pernambuco & $*$ & $*$ & $*$ & $*$ & $*$ & $*$ & 1 & 0.1 & 0.1 & 1 & 0.1 & 0.1 \\
\hline Alagoas & $*$ & $*$ & $*$ & $*$ & $*$ & $*$ & 0 & 0.1 & 0.1 & $*$ & $*$ & $*$ \\
\hline Sergipe & 0 & 0.2 & 0.2 & 0 & 0.2 & 0.2 & $*$ & $*$ & $*$ & $*$ & * & $*$ \\
\hline Bahia & 2 & 0.2 & 0.2 & 2 & 0.2 & 0.2 & 1 & 0.1 & 0.1 & 1 & 0.1 & 0.1 \\
\hline Southeast & 87 & 1.3 & 1.1 & 79 & 1.1 & 1.1 & 78 & 1.0 & 1.1 & 74 & 0.9 & 1.1 \\
\hline Minas Gerais & 22 & 1.3 & 1.2 & 18 & 1.0 & 1.0 & 19 & 1.0 & 1.1 & 24 & 1.2 & 1.5 \\
\hline Espírito Santo & 6 & 2.0 & 1.7 & 3 & 0.9 & 0.9 & 5 & 1.5 & 1.6 & 3 & 0.7 & 0.9 \\
\hline Rio de Janeiro & 10 & 0.7 & 0.6 & 8 & 0.6 & 0.6 & 6 & 0.4 & 0.4 & 11 & 0.7 & 0.8 \\
\hline São Paulo & 50 & 1.4 & 1.3 & 50 & 1.3 & 1.4 & 48 & 1.2 & 1.3 & 37 & 0.9 & 1.1 \\
\hline South & 49 & 2.0 & 1.8 & 45 & 1.8 & 1.8 & 38 & 1.5 & 1.5 & 36 & 1.3 & 1.6 \\
\hline Paraná & 30 & 3.2 & 2.9 & 25 & 2.7 & 2.7 & 25 & 2.5 & 2.6 & 19 & 1.8 & 2.2 \\
\hline Santa Catarina & 7 & 1.4 & 1.2 & 6 & 1.2 & 1.2 & 4 & 0.7 & 0.7 & 4 & 0.6 & 0.7 \\
\hline Rio Grande do Sul & 12 & 1.2 & 1.1 & 13 & 1.3 & 1.3 & 9 & 0.9 & 0.9 & 14 & 1.2 & 1.4 \\
\hline Central West & 18 & 1.6 & 1.4 & 26 & 2.2 & 2.2 & 26 & 2.1 & 2.3 & 17 & 1.3 & 1.5 \\
\hline Mato Grosso do Sul & 4 & 2.0 & 1.8 & 5 & 2.3 & 2.3 & 3 & 1.5 & 1.6 & 3 & 1.1 & 1.3 \\
\hline Mato Grosso & 10 & 4.2 & 3.6 & 14 & 5.6 & 5.6 & 17 & 6.3 & 6.7 & 8 & 2.8 & 3.3 \\
\hline Goiás & 4 & 0.8 & 0.7 & 6 & 1.3 & 1.3 & 5 & 0.9 & 1.0 & 6 & 1.0 & 1.1 \\
\hline Brasilia Federal Distric & ct 0 & 0.2 & 0.2 & 1 & 0.3 & 0.3 & 1 & 0.6 & 0.7 & 1 & 0.2 & 0.2 \\
\hline Brazil & 171 & 1.1 & 1.0 & 173 & 1.0 & 1.0 & 175 & 1.0 & 1.0 & 148 & 0.8 & 0.9 \\
\hline
\end{tabular}

$a$ : preliminary data for 2006. Population adjusted per age having as reference Brazilian 2000 population. Excluded one death without age information in the period of 1996-1998 and one death in the 2002-2004 period. The sum of parts may differ from the total due to number rounding of partial data. TBM: crude rate of mortality; TPM: standardized rate of mortality adjusted by age; $0,0.0,0.00$ : numerical data equal to zero resulting from number rounding of data originally positive. Asterisks means numerical data equal to zero not resulting from number rounding.

In Table II, the crude and standardised mortality rates related to paracoccidioidomycosis by region, state and period are shown. The highest mortality indexes were found in the Southeastern region, especially in the states of São Paulo (SP), Minas Gerais and Rio de Janeiro (RJ), and in the Southern region, in the states of Paraná (PR) and Rio Grande do Sul (Table II).

Cryptococcosis was predominant in the Southeastern and Southern regions. SP and RJ had the highest concentrations of deaths associated with this mycosis (Table III).

There were high rates of mortality due to candidiasis in the Southeast and Northeast of Brazil, with an evident concentration in SP and an increasing number of cases in RJ (Table IV). In the case of histoplasmosis, a low incidence of this mycosis was observed in almost all regions (Table V).

Mortality due to systemic mycoses by age and sexSome mycoses, like paracoccidioidomycosis, had a higher incidence in men than in women, with a ratio of 13:1 (Restrepo et al. 2008), probably as a consequence of hormonal differences (Restrepo et al. 1984). However, this systemic mycosis decreased from $75.3-70.5 \%$ as a cause of death in men over the course of the study period. In contrast, the mortality in women increased from 24.6- 


\section{TABLE III}

Mortality rate TBM and TPM by cryptococcosis (underlying cause codes B45.0-B45.9 - Chapter I - some parasitic and infectious disease - ICD 10) in states and regions of Brazil. Period 1996-2006 ${ }^{a}$

\begin{tabular}{|c|c|c|c|c|c|c|c|c|c|c|c|c|}
\hline \multirow{2}{*}{$\frac{\text { Period }}{\text { Regions and states }}$} & \multicolumn{3}{|c|}{$\begin{array}{c}\text { Average } \\
\text { 1996-1998 (I) }\end{array}$} & \multicolumn{3}{|c|}{$\begin{array}{c}\text { Average } \\
\text { 1999-2001 (II) }\end{array}$} & \multicolumn{3}{|c|}{$\begin{array}{c}\text { Average } \\
\text { 2002-2004 (III) }\end{array}$} & \multicolumn{3}{|c|}{$\begin{array}{c}\text { Average } \\
2005-2006^{a}(\mathrm{IV})\end{array}$} \\
\hline & deaths & TBM & TPM & deaths & TBM & TPM & deaths & TBM & TPM & deaths & TBM & TPM \\
\hline North & 7 & 0.63 & 0.56 & 6 & 0.44 & 0.44 & 8 & 0.58 & 0.54 & 9 & 0.57 & 0.67 \\
\hline Rondônia & $*$ & $*$ & $*$ & $*$ & $*$ & $*$ & 0 & 0.23 & 0.22 & 1 & 0.65 & 0.86 \\
\hline Acre & $*$ & $*$ & * & * & $*$ & $*$ & $*$ & $*$ & $*$ & $*$ & $*$ & $*$ \\
\hline Amazonas & 2 & 0.68 & 0.58 & 1 & 0.36 & 0.36 & 3 & 0.88 & 0.82 & 3 & 0.76 & 0.89 \\
\hline Roraima & 0 & 1.31 & 1.03 & $*$ & $*$ & $*$ & 0 & 0.93 & 0.85 & $*$ & $*$ & $*$ \\
\hline Para & 5 & 0.83 & 0.75 & 4 & 0.71 & 0.71 & 4 & 0.66 & 0.62 & 5 & 0.71 & 0.81 \\
\hline Amapá & 1 & 1.66 & 1.38 & $*$ & $*$ & $*$ & $*$ & $*$ & $*$ & $*$ & $*$ & $*$ \\
\hline Tocantins & $*$ & $*$ & $*$ & 0 & 0.29 & 0.29 & 0 & 0.27 & 0.25 & $*$ & $*$ & $*$ \\
\hline Northeast & 7 & 0.16 & 0.15 & 9 & 0.20 & 0.20 & 10 & 0.20 & 0.20 & 12 & 0.23 & 0.26 \\
\hline Maranhão & 1 & 0.13 & 0.11 & 1 & 0.24 & 0.24 & 2 & 0.34 & 0.33 & 2 & 0.24 & 0.27 \\
\hline Piauí & 1 & 0.25 & 0.23 & 0 & 0.12 & 0.12 & 1 & 0.34 & 0.33 & 2 & 0.50 & 0.53 \\
\hline Ceara & 1 & 0.14 & 0.14 & $*$ & $*$ & $*$ & 2 & 0.30 & 0.29 & 2 & 0.18 & 0.20 \\
\hline Rio Grande do Norte & 0 & 0.13 & 0.11 & * & $*$ & $*$ & * & $*$ & $*$ & $*$ & $*$ & $*$ \\
\hline Paraíba & $*$ & $*$ & $*$ & 0 & 0.10 & 0.10 & 0 & 0.09 & 0.09 & 1 & 0.14 & 0.15 \\
\hline Pernambuco & 2 & 0.31 & 0.30 & 2 & 0.30 & 0.30 & 1 & 0.12 & 0.12 & 3 & 0.35 & 0.38 \\
\hline Alagoas & 1 & 0.25 & 0.23 & * & $*$ & $*$ & 0 & 0.11 & 0.11 & $*$ & $*$ & $*$ \\
\hline Sergipe & 0 & 0.20 & 0.20 & 0 & 0.19 & 0.19 & 0 & 0.18 & 0.17 & 1 & 0.25 & 0.28 \\
\hline Bahia & 1 & 0.10 & 0.10 & 5 & 0.36 & 0.36 & 3 & 0.20 & 0.19 & 4 & 0.25 & 0.28 \\
\hline Southeast & 46 & 0.68 & 0.63 & 40 & 0.55 & 0.55 & 42 & 0.56 & 0.54 & 47 & 0.59 & 0.68 \\
\hline Minas Gerais & 7 & 0.43 & 0.40 & 4 & 0.21 & 0.21 & 4 & 0.20 & 0.19 & 8 & 0.39 & 0.42 \\
\hline Espírito Santo & 1 & 0.23 & 0.22 & 1 & 0.44 & 0.43 & 1 & 0.21 & 0.20 & 3 & 0.73 & 0.81 \\
\hline Rio de Janeiro & 13 & 0.96 & 0.91 & 14 & 0.96 & 0.95 & 11 & 0.74 & 0.72 & 12 & 0.78 & 0.88 \\
\hline São Paulo & 25 & 0.73 & 0.68 & 21 & 0.57 & 0.57 & 27 & 0.69 & 0.66 & 25 & 0.61 & 0.71 \\
\hline South & 12 & 0.50 & 0.46 & 14 & 0.55 & 0.55 & 15 & 0.56 & 0.54 & 14 & 0.50 & 0.55 \\
\hline Paraná & 6 & 0.69 & 0.63 & 5 & 0.52 & 0.52 & 5 & 0.50 & 0.49 & 5 & 0.44 & 0.47 \\
\hline Santa Catarina & 3 & 0.67 & 0.60 & 3 & 0.50 & 0.50 & 2 & 0.30 & 0.28 & 4 & 0.59 & 0.65 \\
\hline Rio Grande do Sul & 2 & 0.24 & 0.22 & 6 & 0.59 & 0.59 & 8 & 0.76 & 0.74 & 6 & 0.50 & 0.58 \\
\hline Central West & 4 & 0.40 & 0.36 & 7 & 0.63 & 0.63 & 6 & 0.51 & 0.49 & 8 & 0.57 & 0.64 \\
\hline Mato Grosso do Sul & 1 & 0.68 & 0.64 & 3 & 1.45 & 1.45 & 1 & 0.61 & 0.59 & 1 & 0.44 & 0.48 \\
\hline Mato Grosso & 1 & 0.44 & 0.37 & 1 & 0.40 & 0.40 & 1 & 0.50 & 0.48 & 4 & 1.24 & 1.39 \\
\hline Goiás & 2 & 0.36 & 0.31 & 3 & 0.53 & 0.53 & 3 & 0.57 & 0.53 & 2 & 0.35 & 0.40 \\
\hline Brasilia Federal District & ct 0 & 0.18 & 0.16 & 1 & 0.33 & 0.33 & 1 & 0.30 & 0.29 & 1 & 0.42 & 0.49 \\
\hline Brazil & 77 & 0.48 & 0.45 & 76 & 0.45 & 0.45 & 81 & 0.46 & 0.44 & 89 & 0.48 & 0.54 \\
\hline
\end{tabular}

$a$ : preliminary data for 2006. Population adjusted per age having as reference Brazilian 2000 population. Excluded one death without age information in the period of 1996-1998. The sum of parts may differ from the total due to number rounding of partial data. TBM: crude rate of mortality; TPM: standardized rate of mortality adjusted by age; 0, 0.0, 0.00: numerical data equal to zero resulting from number rounding of data originally positive. Asterisks means numerical data equal to zero not resulting from number rounding.

29.5\% (MS 2004, 2005). Individually, the standardised rates of mortality related to paracoccidioidomycosis, histoplasmosis, cryptococcosis and coccidioidomycosis (the latter with a low number of cases) were always higher in men, whereas there was no significant gender difference for the other systemic mycoses (Table VI). There was an increase in the mortality from cryptococcosis among women, from $31.8 \%$ (period 1) to $40.1 \%$ (period 4); there was a reduction in the frequency of deaths among men over the same periods, from 68.2$59.9 \%$ (Table VI).
In relation to age, all systemic mycoses showed the highest frequency in people 20 years of age or older. With paracoccidioidomycosis, the highest number of deaths occurred in patients 30 years or older (Table VII).

Basic cause of death when a systemic mycosis was associated with AIDS - Between 1998-2006, 125,633 patients died of AIDS in Brazil and 5,898 (4.7\%) death declarations mentioned a systemic mycosis as an associate cause of death. Systemic mycoses were mentioned in 46.7 per 1,000 deaths of patients for whom AIDS was 
TABLE IV

Mortality rate TBM and TPM of Candidiasis (underlying cause - codes B37.0-B37.9 - Chapter I - some parasitic and infectious disease - ICD 10) in states and regions of Brazil. Period from 1996-2006 ${ }^{a}$

\begin{tabular}{|c|c|c|c|c|c|c|c|c|c|c|c|c|}
\hline \multirow{2}{*}{$\begin{array}{l}\text { Period } \\
\text { Regions and states }\end{array}$} & \multicolumn{3}{|c|}{$\begin{array}{c}\text { Average } \\
\text { 1996-1998 (I) }\end{array}$} & \multicolumn{3}{|c|}{$\begin{array}{c}\text { Average } \\
\text { 1999-2001 (II) }\end{array}$} & \multicolumn{3}{|c|}{$\begin{array}{c}\text { Average } \\
\text { 2002-2004 (III) }\end{array}$} & \multicolumn{3}{|c|}{$\begin{array}{c}\text { Average } \\
\text { 2005-2006* (IV) }\end{array}$} \\
\hline & deaths & TBM & TPM & deaths & TBM & TPM & deaths & $\mathrm{TBM}$ & TPM & deaths & TBM & TPM \\
\hline North & 1 & 0.12 & 0.10 & 1 & 0.05 & 0.05 & 2 & 0.15 & 0.15 & 3 & 0.17 & 0.19 \\
\hline Rondônia & $*$ & $*$ & $*$ & $*$ & $*$ & $*$ & 0 & 0.23 & 0.24 & 2 & 0.97 & 1.08 \\
\hline Acre & $*$ & $*$ & $*$ & $*$ & $*$ & $*$ & $*$ & $*$ & $*$ & $*$ & $*$ & $*$ \\
\hline Amazonas & 1 & 0.27 & 0.22 & $*$ & $*$ & $*$ & 1 & 0.22 & 0.24 & $*$ & $*$ & $*$ \\
\hline Roraima & $*$ & $*$ & $*$ & $*$ & $*$ & $*$ & $*$ & $*$ & $*$ & $*$ & $*$ & $*$ \\
\hline Para & 0 & 0.06 & 0.05 & 0 & 0.05 & 0.05 & 1 & 0.15 & 0.16 & 1 & 0.14 & 0.16 \\
\hline Amapá & $*$ & $*$ & $*$ & $*$ & $*$ & $*$ & $*$ & $*$ & $*$ & $*$ & $*$ & $*$ \\
\hline Tocantins & 0 & 0.31 & 0.27 & 0 & 0.29 & 0.29 & $*$ & $*$ & $*$ & $*$ & $*$ & $*$ \\
\hline Northeast & 11 & 0.24 & 0.24 & 10 & 0.21 & 0.21 & 14 & 0.29 & 0.30 & 11 & 0.20 & 0.22 \\
\hline Maranhão & $*$ & $*$ & $*$ & 1 & 0.18 & 0.18 & 0 & 0.06 & 0.06 & 3 & 0.41 & 0.44 \\
\hline Piauí & $*$ & $*$ & $*$ & 1 & 0.24 & 0.24 & $*$ & $*$ & $*$ & $*$ & $*$ & $*$ \\
\hline Ceara & 5 & 0.77 & 0.81 & 1 & 0.14 & 0.14 & 5 & 0.60 & 0.65 & 1 & 0.12 & 0.16 \\
\hline Rio Grande do Norte & 1 & 0.26 & 0.25 & 1 & 0.24 & 0.24 & 1 & 0.23 & 0.24 & $*$ & $*$ & $*$ \\
\hline Paraíba & $*$ & $*$ & $*$ & $*$ & $*$ & $*$ & $*$ & $*$ & $*$ & 1 & 0.28 & 0.29 \\
\hline Pernambuco & 0 & 0.04 & 0.04 & 1 & 0.17 & 0.17 & 2 & 0.25 & 0.25 & 2 & 0.24 & 0.25 \\
\hline Alagoas & 0 & 0.13 & 0.13 & 0 & 0.12 & 0.12 & 2 & 0.80 & 0.84 & $*$ & $*$ & $*$ \\
\hline Sergipe & $*$ & $*$ & $*$ & $*$ & $*$ & $*$ & 0 & 0.18 & 0.19 & $*$ & $*$ & $*$ \\
\hline Bahia & 4 & 0.34 & 0.32 & 5 & 0.38 & 0.38 & 4 & 0.30 & 0.31 & 4 & 0.29 & 0.30 \\
\hline Southeast & 18 & 0.26 & 0.24 & 18 & 0.25 & 0.25 & 26 & 0.34 & 0.36 & 30 & 0.37 & 0.43 \\
\hline Minas Gerais & 3 & 0.16 & 0.15 & 2 & 0.13 & 0.13 & 2 & 0.13 & 0.13 & 4 & 0.21 & 0.23 \\
\hline Espírito Santo & 1 & 0.35 & 0.33 & 1 & 0.22 & 0.22 & 1 & 0.41 & 0.43 & 2 & 0.58 & 0.64 \\
\hline Rio de Janeiro & 6 & 0.42 & 0.39 & 4 & 0.30 & 0.30 & 8 & 0.56 & 0.58 & 12 & 0.74 & 0.82 \\
\hline São Paulo & 8 & 0.24 & 0.21 & 11 & 0.30 & 0.30 & 14 & 0.36 & 0.38 & 12 & 0.29 & 0.35 \\
\hline South & 8 & 0.32 & 0.29 & 5 & 0.19 & 0.19 & 6 & 0.23 & 0.24 & 7 & 0.26 & 0.31 \\
\hline Paraná & 2 & 0.18 & 0.16 & 2 & 0.17 & 0.17 & 1 & 0.13 & 0.14 & 3 & 0.24 & 0.30 \\
\hline Santa Catarina & 1 & 0.20 & 0.17 & 1 & 0.25 & 0.25 & 2 & 0.36 & 0.37 & 2 & 0.25 & 0.28 \\
\hline Rio Grande do Sul & 5 & 0.51 & 0.46 & 2 & 0.16 & 0.16 & 3 & 0.25 & 0.26 & 3 & 0.28 & 0.33 \\
\hline Central West & 1 & 0.06 & 0.05 & 2 & 0.20 & 0.20 & 3 & 0.24 & 0.26 & 5 & 0.34 & 0.40 \\
\hline Mato Grosso do Sul & $*$ & $*$ & $*$ & 1 & 0,32 & 0,32 & 1 & 0,46 & 0,48 & 1 & 0,22 & 0,24 \\
\hline Mato Grosso & $*$ & $*$ & $*$ & 0 & 0.13 & 0.13 & $*$ & $*$ & $*$ & 1 & 0.35 & 0.39 \\
\hline Goiás & 0 & 0.07 & 0.06 & 1 & 0.13 & 0.13 & $*$ & $*$ & $*$ & 3 & 0.44 & 0.52 \\
\hline Brasilia Federal District & ct 0 & 0.18 & 0.13 & 1 & 0.33 & 0.33 & 2 & 0.91 & 0.98 & 1 & 0.21 & 0.24 \\
\hline Brazil & 38 & 0.24 & 0.22 & 36 & 0.21 & 0.21 & 51 & 0.29 & 0.30 & 54 & 0.29 & 0.33 \\
\hline
\end{tabular}

$a$ : preliminary data for 2006. Population adjusted per age having as reference Brazilian 2000 population. Excluded one death without age information in the period of 1996-1998. The sum of parts may differ from the total due to number rounding of partial data. TBM: crude rate of mortality. TPM: standardized rate of mortality adjusted by age; 0, 0.0, 0.00: numerical data equal to zero resulting from number rounding of data originally positive. Asterisks means numerical data equal to zero not resulting from number rounding.

the underlying cause of death. Cryptococcosis was mentioned in $50.9 \%$ of AIDS death declarations, followed by candidiasis in $30.2 \%$ and histoplasmosis in $10.1 \%$ of cases. Paracoccidioidomycosis, coccidioidomycosis and zygomycosis were less frequent (Table VIII).

Quality of mortality information - For the analysis of mortality data, particularly the cause of death, we observed that there are regions and states that not only provided underestimated numbers of death events, but also that there were doubts concerning the quality of the information. Specifically, the poor quality of informa- tion on the cause of death was reflected in the significant number of records in which the underlying cause of death was not declared. Over the course of the study period, the proportion of such records decreased from $15-10.2 \%$ in the whole country, but there were large differences between states and regions. Records from the Central West, the Southeast and the South showed the lowest percentages of ill-defined records, which decreased during the study period from $10.6-5 \%$ in the Central West, $8.1-5.6 \%$ in the South and $9.4-7.9 \%$ in the Southeast. In the North and Northeast, notwithstand- 
TABLE V

Mortality rate TBM and TPM by histoplasmosis (underlying cause - codes B39.0-B39.9 - Chapter I - some parasitic and infectious disease - ICD 10) in states and regions of Brazil. Period from 1996-2006 ${ }^{a}$

\begin{tabular}{|c|c|c|c|c|c|c|c|c|c|c|c|c|}
\hline \multirow{2}{*}{$\frac{\text { Period }}{\text { Regions and states }}$} & \multicolumn{3}{|c|}{$\begin{array}{c}\text { Average } \\
1996-1998(\mathrm{I})\end{array}$} & \multicolumn{3}{|c|}{$\begin{array}{c}\text { Average } \\
1999-2001 \text { (II) }\end{array}$} & \multicolumn{3}{|c|}{$\begin{array}{c}\text { Average } \\
\text { 2002-2004 (III) }\end{array}$} & \multicolumn{3}{|c|}{$\begin{array}{c}\text { Average } \\
2005-2006^{a}(\mathrm{IV})\end{array}$} \\
\hline & deaths & TBM & TPM & deaths & TBM & TPM & deaths & TBM & TPM & deaths & TBM & TPM \\
\hline North & 1 & 0.12 & 0.10 & * & $*$ & $*$ & 1 & 0.10 & 0.10 & 1 & 0.07 & 0.08 \\
\hline Rondônia & 0 & 0.27 & 0.22 & $*$ & $*$ & $*$ & 0 & 0.23 & 0.24 & $*$ & $*$ & $*$ \\
\hline Acre & $*$ & $*$ & $*$ & $*$ & $*$ & $*$ & $*$ & $*$ & $*$ & $*$ & $*$ & $*$ \\
\hline Amazonas & 0 & 0.14 & 0.12 & $*$ & $*$ & $*$ & $*$ & $*$ & $*$ & $*$ & $*$ & $*$ \\
\hline Roraima & $*$ & $*$ & $*$ & $*$ & $*$ & $*$ & * & $*$ & $*$ & $*$ & $*$ & $*$ \\
\hline Para & 0 & 0.06 & 0.05 & $*$ & $*$ & $*$ & 1 & 0.10 & 0.11 & 1 & 0.14 & 0.16 \\
\hline Amapá & $*$ & $*$ & $*$ & $*$ & $*$ & $*$ & $*$ & $*$ & $*$ & $*$ & $*$ & $*$ \\
\hline Tocantins & 0 & 0.31 & 0.27 & $*$ & $*$ & $*$ & 0 & 0.27 & 0.29 & $*$ & $*$ & $*$ \\
\hline Northeast & 3 & 0.07 & 0.07 & 2 & 0.05 & 0.05 & 2 & 0.05 & 0.05 & 3 & 0.06 & 0.06 \\
\hline Maranhão & $*$ & $*$ & $*$ & $*$ & $*$ & $*$ & $*$ & $*$ & $*$ & $*$ & $*$ & $*$ \\
\hline Piauí & $*$ & $*$ & $*$ & $*$ & $*$ & $*$ & $*$ & $*$ & $*$ & $*$ & $*$ & $*$ \\
\hline Ceara & 2 & 0.29 & 0.26 & 1 & 0.18 & 0.18 & 1 & 0.17 & 0.18 & 2 & 0.18 & 0.20 \\
\hline Rio Grande do Norte & $*$ & $*$ & $*$ & $*$ & $*$ & $*$ & 0 & 0.12 & 0.12 & $*$ & $*$ & $*$ \\
\hline Paraíba & $*$ & $*$ & * & $*$ & $*$ & $*$ & $*$ & $*$ & $*$ & 1 & 0.14 & 0.14 \\
\hline Pernambuco & $*$ & $*$ & $*$ & 0 & 0.04 & 0.04 & 0 & 0.04 & 0.04 & 1 & 0.06 & 0.06 \\
\hline Alagoas & $*$ & $*$ & $*$ & $*$ & $*$ & $*$ & $*$ & $*$ & $*$ & $*$ & $*$ & $*$ \\
\hline Sergipe & $*$ & $*$ & $*$ & $*$ & $*$ & $*$ & $*$ & $*$ & $*$ & $*$ & $*$ & * \\
\hline Bahia & 1 & 0.10 & 0.10 & 1 & 0.05 & 0.05 & 0 & 0.02 & 0.03 & 1 & 0.04 & 0.04 \\
\hline Southeast & 7 & 0.11 & 0.10 & 4 & 0.05 & 0.05 & 4 & 0.05 & 0.05 & 7 & 0.08 & 0.10 \\
\hline Minas Gerais & 3 & 0.16 & 0.15 & 1 & 0.04 & 0.04 & 1 & 0.05 & 0.06 & 1 & 0.03 & 0.03 \\
\hline Espírito Santo & 0 & 0.12 & 0.10 & $*$ & $*$ & $*$ & $*$ & $*$ & $*$ & 1 & 0.15 & 0.23 \\
\hline Rio de Janeiro & 1 & 0.07 & 0.07 & 2 & 0.14 & 0.14 & $*$ & $*$ & $*$ & 3 & 0.16 & 0.19 \\
\hline São Paulo & 3 & 0.10 & 0.08 & 1 & 0.03 & 0.03 & 3 & 0.07 & 0.07 & 3 & 0.07 & 0.09 \\
\hline South & 2 & 0.08 & 0.08 & 1 & 0.05 & 0.05 & 2 & 0.09 & 0.09 & 4 & 0.13 & 0.15 \\
\hline Paraná & 0 & 0.04 & 0.04 & 1 & 0.10 & 0.10 & 1 & 0.10 & 0.10 & 2 & 0.19 & 0.21 \\
\hline Santa Catarina & 0 & 0.07 & 0.05 & $*$ & $*$ & $*$ & 1 & 0.12 & 0.12 & $*$ & $*$ & $*$ \\
\hline Rio Grande do Sul & 1 & 0.14 & 0.12 & 0 & 0.03 & 0.03 & 1 & 0.06 & 0.07 & 2 & 0.14 & 0.17 \\
\hline Central West & 1 & 0.12 & 0.11 & 2 & 0.20 & 0.20 & 3 & 0.22 & 0.23 & 5 & 0.34 & 0.38 \\
\hline Mato Grosso do Sul & 0 & 0.17 & 0.16 & $*$ & $*$ & $*$ & $*$ & $*$ & $*$ & 2 & 0.66 & 0.72 \\
\hline Mato Grosso & $*$ & $*$ & $*$ & 1 & 0.40 & 0.40 & 1 & 0.25 & 0.27 & 1 & 0.18 & 0.20 \\
\hline Goiás & 1 & 0.22 & 0.19 & 1 & 0.20 & 0.20 & 2 & 0.38 & 0.40 & 3 & 0.44 & 0.50 \\
\hline Brasilia Federal District & ct $*$ & $*$ & $*$ & 0 & 0.16 & 0.16 & * & $*$ & $*$ & $*$ & $*$ & $*$ \\
\hline Brazil & 15 & 0.10 & 0.09 & 10 & 0.06 & 0.06 & 12 & 0.07 & 0.07 & 19 & 0.10 & 0.11 \\
\hline
\end{tabular}

$a$ : preliminary data for 2006. Population adjusted per age having as reference Brazilian 2000 population. Excluded one death without age information in the period of 1999-2001. The sum of parts may differ from the total due to number rounding of partial data. TBM: crude rate of mortality; TPM: standardized rate of mortality adjusted by age; 0, 0.0, 0.00: numerical data equal to zero resulting from number rounding of data originally positive. Asterisks means numerical data equal to zero not resulting from number rounding.

ing the significant reduction in ill-defined records from $24.3-17.4 \%$ and $31.4-16.8 \%$ respectively, there remained a high percentage of such cases (MS 2004, 2005).

\section{DISCUSSION}

Most data on the incidence of infectious diseases or of particular systemic mycoses in Brazil are not entirely trustworthy. Mortality data in Brazil suffer from under-notification or incorrect completion of DC. These problems occur mainly due to regional differences in low-income population, population living in rural areas and deaths from undetermined causes. Since most sys- temic mycoses do not require obligatory notification to the health system authority, the evaluation of mortality data can be an important epidemiological tool in assessing the real magnitude of systemic mycoses in Brazil's vast territory.

The mortality rate of systemic mycoses included in our study shows that they are the 10th most common cause of death among infectious and parasitic diseases. The death rate is lower than that of hanseniasis, pertussis, sepsis, AIDS, intestinal infections, tuberculosis, diseases caused by protozoan agents such as malaria and Chagas disease, viral hepatitis, sequellae from infec- 
TABLE VI

Numbers and frequency of deaths by gender and mention of systemic mycosis. Brazil, period from 1996-2006 ${ }^{a}$

\begin{tabular}{|c|c|c|c|c|c|c|c|c|}
\hline \multirow[b]{2}{*}{ Mycosis/period } & \multicolumn{2}{|c|}{$\begin{array}{c}\text { Average } \\
\text { 1996-1998 (I) }\end{array}$} & \multicolumn{2}{|c|}{$\begin{array}{c}\text { Average } \\
\text { 1999-2001 (II) }\end{array}$} & \multicolumn{2}{|c|}{$\begin{array}{c}\text { Average } \\
\text { 2002-2004 (III) }\end{array}$} & \multicolumn{2}{|c|}{$\begin{array}{c}\text { Average } \\
2005-2006^{a}(\mathrm{IV})\end{array}$} \\
\hline & $\mathrm{n}$ & $\%$ & $\mathrm{n}$ & $\%$ & $\mathrm{n}$ & $\%$ & $\mathrm{n}$ & $\%$ \\
\hline \multicolumn{9}{|l|}{ Candidiasis } \\
\hline Men & 17 & 44.8 & 22 & 62.0 & 28 & 53.9 & 28 & 51.9 \\
\hline Women & 21 & 55.2 & 14 & 38.0 & 24 & 46.1 & 26 & 48.1 \\
\hline Total & 39 & 100.0 & 36 & 100.0 & 51 & 100,0 & 54 & 100,0 \\
\hline \multicolumn{9}{|c|}{ Coccidioidomycosis } \\
\hline Men & 0 & 33.3 & 1 & 100.0 & 2 & 100.0 & 2 & 80.0 \\
\hline Women & 1 & 66.7 & 0 & 0,0 & 0 & 0,0 & 1 & 20.0 \\
\hline Total & 1 & 100.0 & 1 & 100.0 & 2 & 100.0 & 3 & 100,0 \\
\hline \multicolumn{9}{|l|}{ Histoplasmosis } \\
\hline Men & 12 & 80.4 & 6 & 63.3 & 9 & 70.3 & 12 & 62.2 \\
\hline Women & 3 & 19.6 & 4 & 36.7 & 4 & 29.7 & 7 & 37.8 \\
\hline Total & 15 & 100.0 & 10 & 100.0 & 12 & 100.0 & 19 & 100,0 \\
\hline \multicolumn{9}{|c|}{ Paracoccidioidomycosis + Blastomycosis } \\
\hline Men & 148 & 86.7 & 155 & 89.6 & 156 & 88.8 & 127 & 86.4 \\
\hline Women & 22 & 13.1 & 18 & 10.4 & 20 & 11.2 & 20 & 13.6 \\
\hline Total & 171 & 100.0 & 173 & 100.0 & 175 & 100.0 & 147 & 100,0 \\
\hline \multicolumn{9}{|l|}{ Aspergillosis } \\
\hline Men & 7 & 61.1 & 8 & 65.8 & 13 & 70.9 & 10 & 57.6 \\
\hline Women & 5 & 38.9 & 4 & 34.2 & 5 & 29.1 & 7 & 42.4 \\
\hline Total & 12 & 100.0 & 13 & 100.0 & 18 & 100.0 & 17 & 100.0 \\
\hline \multicolumn{9}{|l|}{ Cryptococcosis } \\
\hline Men & 53 & 68.2 & 51 & 67.0 & 50 & 62,1 & 53 & 59.9 \\
\hline Women & 25 & 31.8 & 25 & 33.0 & 31 & 37,9 & 36 & 40.1 \\
\hline Total & 78 & 100,0 & 76 & 100.0 & 81 & 100.0 & 89 & 100.0 \\
\hline \multicolumn{9}{|l|}{ Zygomycosis } \\
\hline Men & 0 & 20.0 & 2 & 58.3 & 3 & 61.5 & 2 & 50.0 \\
\hline Women & 1 & 80.0 & 2 & 41.7 & 2 & 38.5 & 2 & 50.0 \\
\hline Total & 2 & 100.0 & 4 & 100.0 & & 100.0 & 3 & 100.0 \\
\hline
\end{tabular}

$a$ : preliminary data for 2006. In the period, two deaths were excluded because of unspecified gender.

tious and parasitic diseases and zoonotic diseases such as leptospirosis and brucellosis. Isolated data showed that paracoccidioidomycosis was the main cause of death among systemic mycoses, followed by cryptococcosis, candidiasis and histoplasmosis.

Accordingly, paracoccidioidomycosis was responsible for approximately half of the total deaths due to systemic mycoses, with SP and PR having the highest mortality rates; however, these rates clearly decreased when comparing the first and the last periods of the present study. Previous studies showed similar results when only SP (Santo 2008) or all Brazilian states (Coutinho et al. 2002) were considered. Among other diseases caused by dimorphic fungi, attention should be given to histoplasmosis and coccidioidomycosis, which showed increasing mortality rates. Coccidioidomycosis has been diagnosed in the Northeastern region of Brazil, mainly in the states of Ceará and Piauí (Wanke et al. 1999, Capone et al. 2008). However, the DC were emitted in different regions of Brazil, probably because the affected individuals had been on vacation in endemic areas prior to disease development or looked for a specialised medical centre in places far from their homes. This kind of information is important in order to correlate the real area of infection with the deaths occurring outside that region.

Candidiasis, cryptococcosis and aspergillosis are always associated with immunosuppressed patients (Fidel 1999, Sweeney et al. 2003, Maschmeyer et al. 2007). For these mycoses, the Southeastern region showed the highest mortality rate, followed by the Northeast and South of Brazil. When analysing mentions of any systemic mycosis in individuals who died from AIDS, we found 5,898 such records out of 125,633 deaths. Cryptococcosis was mentioned in $50.9 \%$ of these records, representing 23.9 cases out of every 1,000 AIDS-related deaths, followed by candidiasis with 14.17 , histoplasmosis with 4.73 and aspergillosis with 3.4 records per 1,000 AIDSrelated deaths. These data differ from those obtained in the first step of study, because we considered AIDS as the underlying cause of death and looked for any mention of associated systemic mycosis. 
TABLE VII

Numbers and frequency of deaths by age and mention of systemic mycosis. Brazil, period from 1996-2006 ${ }^{a}$

\begin{tabular}{|c|c|c|c|c|c|c|c|c|c|}
\hline Mycosis & Age & $\begin{array}{c}\text { Average } \\
1996-1998\end{array}$ & $\%$ & $\begin{array}{l}\text { Average } \\
999-2001\end{array}$ & $\%$ & $\begin{array}{c}\text { Average } \\
2002-2004\end{array}$ & $\%$ & $\begin{array}{c}\text { Average } \\
2005-2006^{a}\end{array}$ & $\%$ \\
\hline \multirow[t]{6}{*}{ Candidiasis } & $0-14$ & 14 & 35.7 & 7 & 18.5 & 14 & 27.3 & 7 & 13.0 \\
\hline & $15-19$ & 1 & 1.7 & - & 0.0 & - & 0.6 & - & 0.0 \\
\hline & $20-29$ & 1 & 3.5 & 3 & 7.4 & 4 & 8.4 & 4 & 6.5 \\
\hline & $30-59$ & 11 & 29.6 & 13 & 36.1 & 15 & 29.9 & 16 & 28.7 \\
\hline & 60 or more & 11 & 29.6 & 14 & 38.0 & 17 & 33.8 & 28 & 51.9 \\
\hline & Total & 38 & 100.0 & 36 & 100.0 & 51 & 100.0 & 54 & 100.0 \\
\hline \multirow[t]{6}{*}{ Coccidioidomycosis } & $0-14$ & - & 33.3 & - & - & - & - & - & - \\
\hline & $15-19$ & - & - & - & 33.3 & - & - & - & - \\
\hline & $20-29$ & - & 33.3 & - & - & 1 & 33.3 & - & . \\
\hline & $30-59$ & - & 33.3 & 1 & 66.7 & 1 & 33.3 & 2 & 60.0 \\
\hline & 60 or more & - & - & - & - & 1 & 33.3 & 1 & 40.0 \\
\hline & Total & 1 & 100.0 & 1 & 100.0 & 2 & 100.0 & 3 & 100.0 \\
\hline \multirow[t]{6}{*}{ Histoplasmosis } & $0-14$ & 1 & 6.5 & 1 & 13.8 & - & 0.0 & 1 & 5.4 \\
\hline & $15-19$ & - & - & - & 3.4 & 1 & 5.4 & - & 0.0 \\
\hline & $20-29$ & 3 & 17.4 & 1 & 13.8 & 1 & 8.1 & 3 & 13.5 \\
\hline & $30-59$ & 8 & 54.3 & 4 & 41.4 & 7 & 59.5 & 10 & 51.4 \\
\hline & 60 or more & 3 & 21.7 & 3 & 27.6 & 3 & 27.0 & 6 & 29.7 \\
\hline & Total & 15 & 100.0 & 10 & 100.0 & 12 & 100.0 & 19 & 100.0 \\
\hline \multirow[t]{6}{*}{ Cryptococcosis } & $0-14$ & 3 & 3.4 & 4 & 4.8 & 3 & 3.7 & 5 & 5.6 \\
\hline & $15-19$ & 3 & 3.4 & 1 & 1.3 & 3 & 3.7 & 4 & 4.5 \\
\hline & $20-29$ & 15 & 19.0 & 14 & 18.1 & 11 & 13.6 & 10 & 10.7 \\
\hline & $30-59$ & 46 & 59.5 & 48 & 63.0 & 48 & 59.3 & 58 & 65.5 \\
\hline & 60 or more & 11 & 14.7 & 10 & 12.8 & 16 & 19.8 & 12 & 13.6 \\
\hline & Total & 77 & 100.0 & 76 & 100.0 & 81 & 100.0 & 89 & 100.0 \\
\hline \multirow{5}{*}{ Aspergillosis } & $15-19$ & - & 2.8 & - & - & 1 & 3.6 & - & 0.0 \\
\hline & $20-29$ & 1 & 11.1 & 2 & 13.2 & 1 & 5.5 & 2 & 12.1 \\
\hline & $30-59$ & 5 & 41.7 & 5 & 36.8 & 6 & 34.5 & 7 & 39.4 \\
\hline & 60 or more & 5 & 41.7 & 6 & 44.7 & 9 & 50.9 & 8 & 48.5 \\
\hline & Total & 11 & 97.2 & 12 & 94.7 & 17 & 94.5 & 17 & 100.0 \\
\hline \multirow[t]{6}{*}{ Zygomycosis } & $0-14$ & 0 & 20.0 & 1 & 16.7 & 0 & 7.7 & 1 & 16.7 \\
\hline & $15-19$ & - & - & 0 & 8.3 & 1 & 15.4 & 1 & 16.7 \\
\hline & $20-29$ & - & - & 0 & 8.3 & 1 & 15.4 & 1 & 16.7 \\
\hline & $30-59$ & 1 & 40.0 & 2 & 50.0 & 2 & 46.2 & 1 & 16.7 \\
\hline & 60 or more & 1 & 40.0 & 1 & 16.7 & 1 & 15.4 & 1 & 33.3 \\
\hline & Total & 2 & 100.0 & 4 & 100.0 & 4 & 100.0 & 3 & 100.0 \\
\hline \multirow{6}{*}{$\begin{array}{l}\text { Paracoccidioidomycosis } \\
\text { and } \\
\text { blastomycosis }\end{array}$} & $0-14$ & 4 & 2.1 & 2 & 1.4 & 3 & 1.7 & 2 & 1.4 \\
\hline & $15-19$ & 4 & 2.1 & 2 & 1.2 & 2 & 1.0 & 2 & 1.0 \\
\hline & $20-29$ & 10 & 6.1 & 7 & 3.9 & 6 & 3.2 & 7 & 4.7 \\
\hline & $30-59$ & 90 & 52.9 & 92 & 53.1 & 97 & 55.4 & 77 & 52.2 \\
\hline & 60 or more & 63 & 36.7 & 70 & 40.5 & 68 & 38.7 & 60 & 40.7 \\
\hline & Total & 171 & 100.0 & 173 & 100.0 & 175 & 100.0 & 148 & 100.0 \\
\hline \multirow{6}{*}{$\begin{array}{l}\text { Other } \\
\text { systemic mycosis }\end{array}$} & $0-14$ & 22 & 7.0 & 15 & 4.9 & 21 & 6.2 & 16 & 4.7 \\
\hline & $15-19$ & 7 & 2.3 & 4 & 1.3 & 7 & 2.0 & 6 & 1.8 \\
\hline & $20-29$ & 31 & 9.7 & 26 & 8.4 & 24 & 7.1 & 25 & 7.6 \\
\hline & $30-59$ & 162 & 51.2 & 164 & 52.5 & 177 & 51.3 & 169 & 51.0 \\
\hline & 60 or more & 94 & 29.8 & 102 & 32.8 & 115 & 33.4 & 116 & 34.9 \\
\hline & Total & 316 & 100.0 & 312 & 100.0 & 344 & 100.0 & 331 & 100.0 \\
\hline
\end{tabular}

a: preliminary data for 2006 .

Regarding sex and age, males account for more than $70 \%$ of deaths and most deaths occur in the age range of 30-59 years old, followed by the age range of 60 or more years old. However, some dimorphic mycoses such as paracoccidioidomycosis showed a ratio of 13:1 (men:women) in incidence due to the seemingly natural protection that women have thanks to $\beta$ oestrogen ( $\mathrm{Re}$ strepo et al. 1984, 2008).

In conclusion, our data present the mortality rates associated with the most important systemic mycoses in 
TABLE VIII

Number and frequency of mentions to systemic mycoses in deaths with underlying cause of AIDS in Brazil from 1996-2006 ${ }^{a}$

\begin{tabular}{lccr}
\hline Mention (3C - ICD - 10) & $\mathrm{n}$ & $\%$ & Number per 1,000 deaths of AIDS \\
\hline B45 Cryptococcosis & 3001 & 50.9 & 23.89 \\
B37 Candidiasis & 1780 & 30.2 & 4.17 \\
B39 Histoplasmosis & 594 & 10.1 & 4.73 \\
B44 Aspergillosis & 427 & 7.2 & 3.40 \\
B40 e B41 (Blastomycosis/Paracoccidioidomycosis) & 84 & 1.4 & 0.67 \\
B38 Coccidioidomycosis & 11 & 0.2 & 0.09 \\
B46 Zygomicosis & 1 & 0.0 & 0.01 \\
Total (systemic mycoses) & 5.898 & 100.0 & 46.95
\end{tabular}

$a$ : preliminary data for 2006. Obits by AIDS as basic cause of death (Cod B20-24 - Chapter I - same infectious and parasitic disease - ICD - 10). Mention to systemic mycosis anywhere in part I or II of obit declaration with AIDS as the basic cause of death.

Brazil. Since the real number of patients with systemic mycoses is unknown, the mortality rates can be used as a tool for elucidating the incidence and severity of each specific disease. It is important to point out that immunosuppressive diseases like AIDS can "hide" the simultaneous presence of systemic mycoses and, consequently, their epidemiological relevance.

\section{REFERENCES}

Capone D, Marchiori E, Wanke B, Dantas KE, Cavalcanti MA, Deus Filho A, Escuissato DL, Warszawiak D 2008. Acute pulmonary coccidioidomycosis: CT findings from 15 patients. Br J Radiol 81: $721-724$.

Coutinho ZF, da Silva D, Lazéra M, Petri V, Oliveira RM, Sabroza PC, Wanke B 2002. Paracoccidioidomycosis mortality in Brazil (1980-1995). Cad Saude Publica 18: 1441-1454.

Fidel PL Jr 1999. Host defense against oropharyngeal and vaginal candidiasis: site-specific differences. Rev Iberoam Micol 16: 8-15.

IBGE - Fundação Instituto Brasileiro de Geografia e Estatística 2000. Censos (2000), contagem (1996) e projeções intercensitárias. Available from: www.ibge.gov.br.

Laurenti R, Mello Jorge MHP, Lebrão ML, Gotlieb SLD, Almeida MF 2005. Estatísticas vitais: contando os nascimentos e as mortes. Rev Bras Epidemiol 8: 108-110.

Maschmeyer G, Haas A, Cornely OA 2007. Invasive aspergillosis: epidemiology, diagnosis and management in immunocompromised patients. Drugs 67: 1567-1601.

MS - Ministério da Saúde. Secretaria de Vigilância em Saúde. Departamento de Análise de Situação de Saúde 2004. Saúde Brasil 2004 - uma análise da situação de saúde no Brasil, Brasília (DF), 364 pp.
MS - Ministério da Saúde. Secretaria de Vigilância em Saúde. Departamento de Análise de Situação em Saúde 2005. Saúde Brasil 2005 - uma análise da situação de saúde no Brasil, Brasília (DF), 882 pp.

MS - Ministério da Saúde 2005. Departamento de Informática do SUS - DATASUS 1979 a 2005. Arquivos eletrônicos dos dados do Sistema de Informações sobre Mortalidade - SIM. [Base de dados 1996 a 2005]. Available from: www.datasus.gov.br.

Restrepo A, Benard G, Castro CC, Agudelo CA, Tabón AM 2008. Pulmonary paracoccidioidomycosis. Semin Respir Crit Care Med 29: 182-197.

Restrepo A, Salazar ME, Cano LE, Stover EP, Feldman D, Stevens DA 1984. Estrogens inhibit mycelium to yeast transformation in the fungus Paracoccidioides brasiliensis: implications for resistance of females to paracoccidioidomycosis. Infect Immun 46: 346-353.

Rumel D 1988. Razões de mortalidade frente ao efeito desigualdade em estudos de mortalidade associada a categorias ocupacionais e níveis sociais. Rev Saude Publica 22: 335-340.

Santo AH 2008. Tendência da mortalidade relacionada à paracoccidioidomicose, Estado de São Paulo, Brasil, 1985 a 2005: estudo usando causas múltiplas de morte. Rev Panam Salud Publica 23: 313-324.

Sweeney DA, Caserta MT, Korones DN, Casadevall A, Goldman D 2003. A ten-year-old boy with a pulmonary nodule secondary to Cryptococcus neoformans: case report and review of the literature. Pediatr Infect Dis J 22: 1089-1093.

Wanke B, Lazera M, Monteiro PCF, Lima FC, Leal MJ, Ferreira Filho PL, Kaufman L, Pinner RW, Ajello L 1999. Investigation of an outbreak of endemic coccidioidomycosis in Brazil's Northeastern state of Piauí with a review of the occurrence and distribution of Coccidioides immitis in three other Brazilian states. Mycopathologia 148: 57-67. 